\title{
SCALABLE FAULT-TOLERANT LOCATION MANAGEMENT SCHEME FOR MOBILE IP
}

\author{
JinHo Ahn, Sung-Gi Min and ChongSun Hwang \\ Dept. of Computer Science and Engineering, Korea University \\ 5-1 Anam-dong, Sungbuk-gu, Seoul 136-701, Republic of Korea
}

\begin{abstract}
As the number of mobile nodes registering with a network rapidly increases in Mobile IP, multiple mobility (home or foreign) agents can be allocated to a network in order to improve performance and availability. Previous fault-tolerant schemes (denoted by PRT schemes) to mask failures of the mobility agents use passive replication techniques. However, they result in high failure-free latency during registration process if the number of mobility agents in the same network increases, and force each mobility agent to manage bindings of all the mobile nodes registering with its network. In this paper, we present a new fault-tolerant scheme (denoted by CML scheme) using checkpointing and message logging techniques. The CML scheme achieves low failure-free latency even if the number of mobility agents in a network increases, and improves scalability to a large number of mobile nodes registering with each network compared with the PRT schemes. Additionally, the CML scheme allows each failed mobility agent to recover bindings of the mobile nodes registering with the mobility agent when it is repaired even if all the other mobility agents in the same network concurrently fail.
\end{abstract}

\section{INTRODUCTION}

A Mobile IP based system extends an IP based distributed system to support mobility of nodes by providing Mobile Nodes (MNs) with continuous network connections while changing their locations [Ghosh (1998), Johnson (1994), Perkins (1996b), Solomon (1998)]. In other words, it transparently provides mobility for nodes while backward compatible with the current IP routing scheme by using two kinds of Mobility Agents (MAs), home agent and foreign agent. In the system, each home agent must maintain information about the current careof address of each $\mathrm{MN}$ registering with the home agent and forward packets, destined to the MN, to the care-of address for the MN. Each foreign agent should offer a care-of address for each visiting MN and forward packets to the MN. If each MN uses a collocated care-of address, it must have the same function of a foreign agent.

As mobile computing is increasingly gaining popularity, MAs should serve a large number of MNs. Thus, they may be single points of failure and potential performance bottlenecks. Especially, if a home agent fails, all the MNs served by it can't communicate with other nodes. The problems can be solved by allowing multiple MAs to be assigned to the same network. Previous fault-tolerant schemes (denoted by PRT schemes) [Ghosh (1998)] to mask failures of MAs in Mobile IP use passive replication techniques. In the PRT schemes, each MA must always maintain bindings of all MNs registering with its network. Moreover, if each MA receives a registration request message from a MN in these schemes, it should process the message, forward the message to its peers and then wait until it has received all the acknowledgement messages from them. Thus, the PRT schemes result in high failure-free overhead during registration process if the number of MAs in the same network increases and are not scalable to the number of MNs registering with each network. Log-based rollback recovery schemes, which use checkpointing and message logging techniques, are inexpensive during failure-free operation compared with the schemes using passive replication techniques [Alvisi (1993)]. In this paper, we present a new fault-tolerant scheme (denoted by CML scheme) for MAs using checkpointing and message logging techniques. The CML scheme reduces the failure-free latency even if the number of MAs in a network increases, and improves scalability to a large number of MNs registering with each network compared with the PRT schemes and provides fast recovery for taking over failed MAs. Additionally, the CML scheme allows each failed MA to recover bindings of the MNs registering with the MA when it is repaired even if all the MAs in the same network fail.

The rest of the paper is organized as follows. In section 2, we describe the overview of Mobile IP based Systems and problems of the previous fault-tolerant schemes respectively. In section 3, we present our fault-tolerant scheme, explain the description of the scheme in details and prove its correctness. Section 4 compares our scheme with others and then, in section 5 , we conclude this paper.

\section{PRELIMINARIES}

\section{System Model}

In Mobile IP, each MN must have a unique home address and a home agent on its home network. If a MN moves from its home network to a foreign network, the current location of the MN is identified as a care-of address (COA), and the mapping between the home address and the COA of the MN is called a binding [Solomon (1998)]. Whenever the MN enters into a new foreign network, it must register by sending a registration request message to a foreign agent (FA) in the new network. The request message includes the home address of the MN 
and the $\mathrm{IP}$ address of its home agent (HA). Then, the FA sends a registration request message to the HA. The message contains the home address and COA of the MN, the IP address of the HA, a registration lifetime and an identifier which uniquely identifies the registration request message. When the HA receives the request message, it updates the binding of the MN, and then sends a registration reply message back to the FA. When the FA receives the reply message, it updates its own table and forwards the message to the MN. A MN in a foreign network can obtain a COA in one of two ways as follows. First, if there is a FA in the foreign network, the MN will attempt to obtain a care-of address from the agent by using an agent discovery protocol [Johnson (1994), Solomon (1998)]. In this case, the IP address of the FA is used as the COA of the node. Second, if there is no FA in the network, the MN can obtain a collocated COA in the network using a DHCP-like protocol [Droms (1993)]. If a correspondent node (CN), which may be a MN or a FN, sends a packet to a MN, this packet is routed to the home network of the $\mathrm{MN}$ when the $\mathrm{MN}$ is in the network. When the $\mathrm{MN}$ is not in its home network, its HA intercepts, encapsulates and then tunnels the packet to the FA in the foreign network where the MN is currently located [Perkins (1996a), Perkins (1996c), Simpson (1995)]. Then, the FA de-tunnels the packet to the MN. If the MN currently uses a collocated COA, de-capsulation of the packet must be carried out by the MN rather than the FA. However, this triangle routing scheme may be inefficient because the messages, destined to each MN, should be first routed to its HA. In order to solve the problem, the route optimization scheme [Perkins (1996d)] was proposed in which the messages are routed directly to the COA of the MN. In this scheme, each $\mathrm{CN}$ maintains a binding cache containing the COAs of the communicating MNs. The drawback of the scheme is that it forces the $\mathrm{CN}$ to be aware of mobility of the MNs. Each FA or HA is connected by a fixed wired network, which provides reliable FIFO delivery of messages. Each MN can directly communicate with its local FA or HA via a reliable FIFO wireless network only if the MN is in the network covered by the mobility agent. We assume that nodes, including FAs, HAs, other FNs and MNs, fail, in which case they lose the contents of their volatile memories and stop their executions, according to the fail stop model [Schlichting (1985)]. Multiple FAs or HAs can be allocated to each network in order to serve a large number of MNs on the network like in Figure 1 [Binkley (1997)]. Separate nodes or a single node on a network may have the function of HA and FA. Similarly, the existing IP routers or separate nodes on a network may implement either function or both.

We assume that the communication network is immune to partitioning and there is a stable storage that every MA can always access that persists beyond processor failures, thereby supporting recovery from failure of an arbitrary number of processors [Elnozahy (1992)]. The execution of each MA is piecewise deterministic [Elnozahy (1999), Strom (1985)]: At any point during the execution, a state interval of the MA is determined by a non-deterministic event such as delivering a registration request message. The $k$-th state interval of a MA $p$, denoted by $s i_{p}{ }^{k}(k>0)$, is started by the delivery event of the $k$-th registration request message $m$ of $p$, denoted by $\operatorname{dev}_{p}{ }^{k}(m)$. Let $p$ 's state, $s_{p}{ }^{i}=\left\{s i_{p}{ }^{0}, s i_{p}{ }^{\prime}, \ldots, s i_{p}{ }^{i}\right\}$, represent the sequence of all state intervals up to $s i_{p}{ }^{i}$. Therefore, given $p$ 's initial state, $s i_{p}{ }^{0}$, and the non-deterministic events, $\left[\operatorname{dev}_{p}{ }^{1}, \operatorname{dev}_{p}{ }^{2}, \ldots, \operatorname{dev}_{p}{ }^{i}\right]$, its corresponding state $s_{p}{ }^{l}$ is uniquely determined. All information needed for replaying the delivery event of a message during recovery is called determinant of the event.

Definition 1. $s i_{p}^{i}$ is stable if a determinant of $d e v_{p}^{i}(m)$ is saved on stable storage.

Definition 2. $s_{p}{ }^{i}$ is volatile if it is not stable.

Definition 3. $s_{p}^{i}$ is recoverable if the system has sufficient information for replaying its failure-free execution up to $s i_{p}^{i}$ in any future failures.

Lemma 1. $s i_{p}{ }^{i}$ is stable if $s_{p}{ }^{i}$ is recoverable.

Proof. We prove this lemma by contradiction. Assume that $s i_{p}{ }^{i}$ is unstable, i.e., volatile if $s_{p}{ }^{i}$ is recoverable. $p$ can replay $\operatorname{dev}_{p}{ }^{i}(m)$ in any failure by Definition 3. To do so, it has to obtain the determinant of $\operatorname{dev}_{p}^{i}(m)$ during recovery. The stable storage is the only one that survives in an arbitrary number of failures by the fail-stop model and the property of the storage. Thus, the determinant should have been saved on stable storage in a previous failure free execution. Therefore, $s i_{p}{ }^{i}$ is stable by Definition 1 . Hence, this contradicts the hypothesis. 


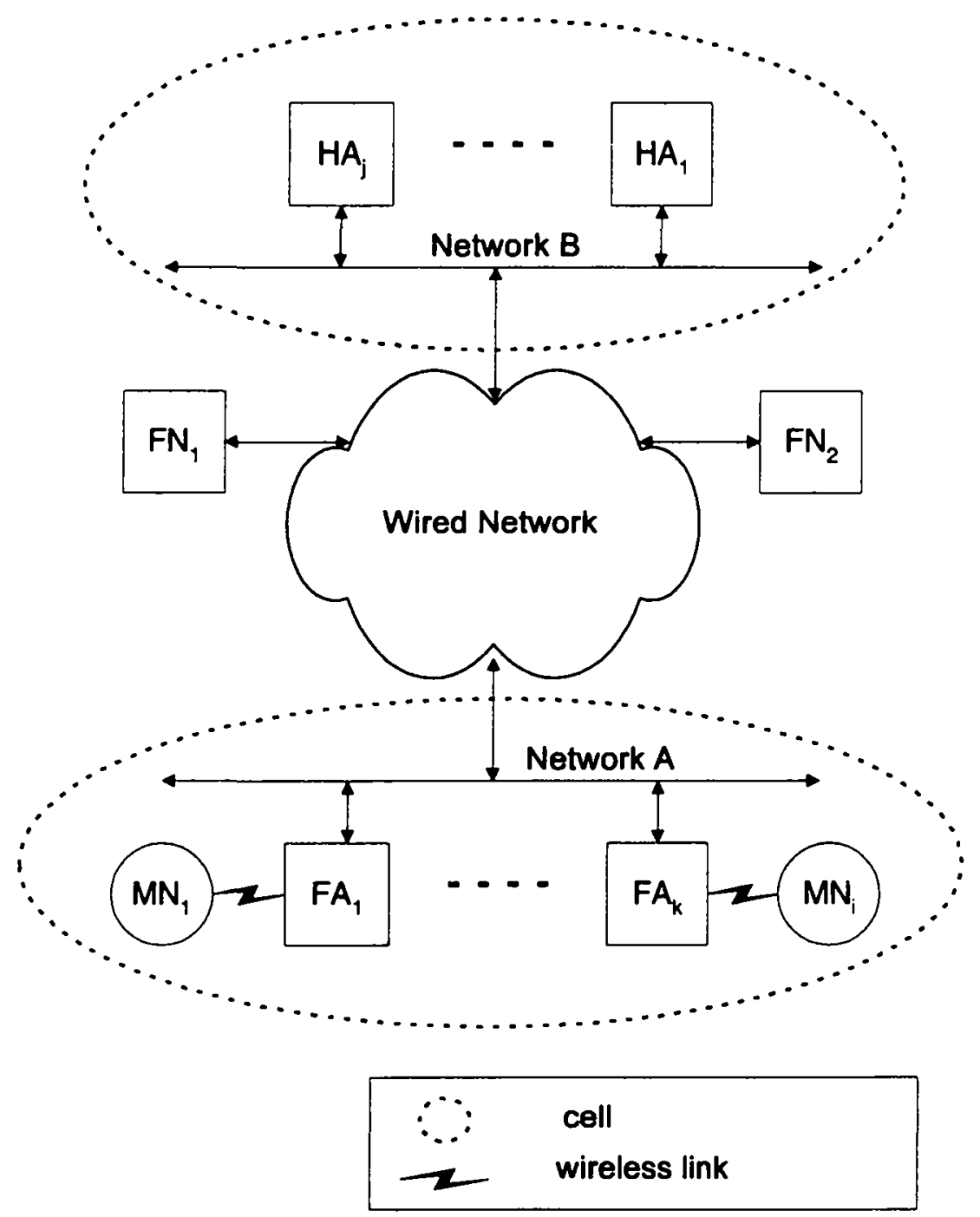

Figure 1 Mobile IP Infrastructure

Lemma 2. $s_{p}{ }^{\prime}$ is recoverable if $\forall k(0 \leq k \leq i): s i_{p}{ }^{k}$ is stable.

Proof. The proof proceeds by induction on $i$, the index of the state interval of each mobility agent $p$.

[Base case]

If $s i_{p}{ }^{0}$ is stable in this case, $s_{p}{ }^{0}$ is trivially recoverable because $s i_{p}{ }^{0}$ is the initial state interval of $p$ and deterministic.

\section{[Induction hypothesis]}

We assume that the theorem is true in case that $i=n$.

[Induction step]

If $s_{p}{ }^{n}$ is recoverable and there is the determinant of $\operatorname{dev}_{p}{ }^{n+1}(m)$ on stable storage, the theorem is true for $p$ in case that $i=n+1$. In this step, $s_{p}{ }^{n}$ is recoverable by induction hypothesis because $\forall k(0 \leq k \leq i):\left(s i_{p}{ }^{k}\right.$ is stable), and $s i_{p}{ }^{n+1}$ is stable. Therefore, $s_{p}{ }^{n+1}$ is recoverable.

By the induction, $s_{p}{ }^{i}$ is recoverable if $\forall k(0 \leq k \leq i): s i_{p}{ }^{k}$ is stable.

\section{Problems of PRT Schemes}

PRT Schemes [Ghosh (1998)] to mask failures of multiple MAs in a network use passive replication techniques. In the PRT schemes, each MA in the network must always maintain bindings of all MNs registering with the network. Moreover, if each MA receives a registration request message from a $\mathrm{MN}$ in the schemes, it should process the message and forward the message to its peers and wait until it has received all the acknowledgement messages from them. Thus, the PRT schemes result in high failure-free latency during registration process as the number of MAs allocated to a network increases, and are not scalable to the number of MNs registering with each network. Additionally, in the schemes, each failed MA cannot recover bindings of the MNs registering with the MA when it is repaired if all the MAs in the same network fail. 
To illustrate the stated problems of the schemes, consider the examples shown in Figure 2 and 5 . In Figure 2, there are three MAs, $M A_{1}, M A_{2}$ and $M A_{3}$ in network $A$. If $M A_{1}$ receives a registration request message from a $M N$, named $M A_{l}$, it updates $M A_{l}$ 's binding using the message and then forwards the message to $M A_{2}$ and $M A_{3}$, respectively. After $M A_{2}$ and $M A_{3}$ have received the message from $M A_{1}$, they update $M A_{1}$ 's binding using the message, and then send each an acknowledgement message to $M A_{l}$. After $M A_{t}$ has received the acknowledgement messages from $M A_{2}$ and $M A_{3}$, it sends a registration reply message to $M A_{I}$. In this figure, we can see that the total number of messages generated per registration request message and the number of messages on the critical path for registration operation in the previous schemes increases as the number of MAs allocated to a network increases. Thus, they result in high latency during registration process.

Additionally, in the PRT schemes, each MA in a network should still maintain bindings of all the MAs registering with the network. For examples, in Figure 3, $6000 \mathrm{MNs}$ register with network $A$, and $M A_{I}$ serves 2000 among them, $M A_{2}$ serves 1000 and $M A_{3}$ serves 3000 . However, $M A_{1}, M A_{2}$ and $M A_{3}$ should respectively maintain the bindings of $6000 \mathrm{MNs}$ registering with network $A$. Therefore, we can see that the traditional schemes are not scalable to a large number of MNs registering with the same network even if there are multiple MAs in the network. Moreover, in the schemes, if $M A_{1}, M A_{2}$ and $M A_{3}$ all concurrently fail in Figure 3, each of the three MAs cannot recover bindings of the MNs registering with it from any other MA when it is repaired.

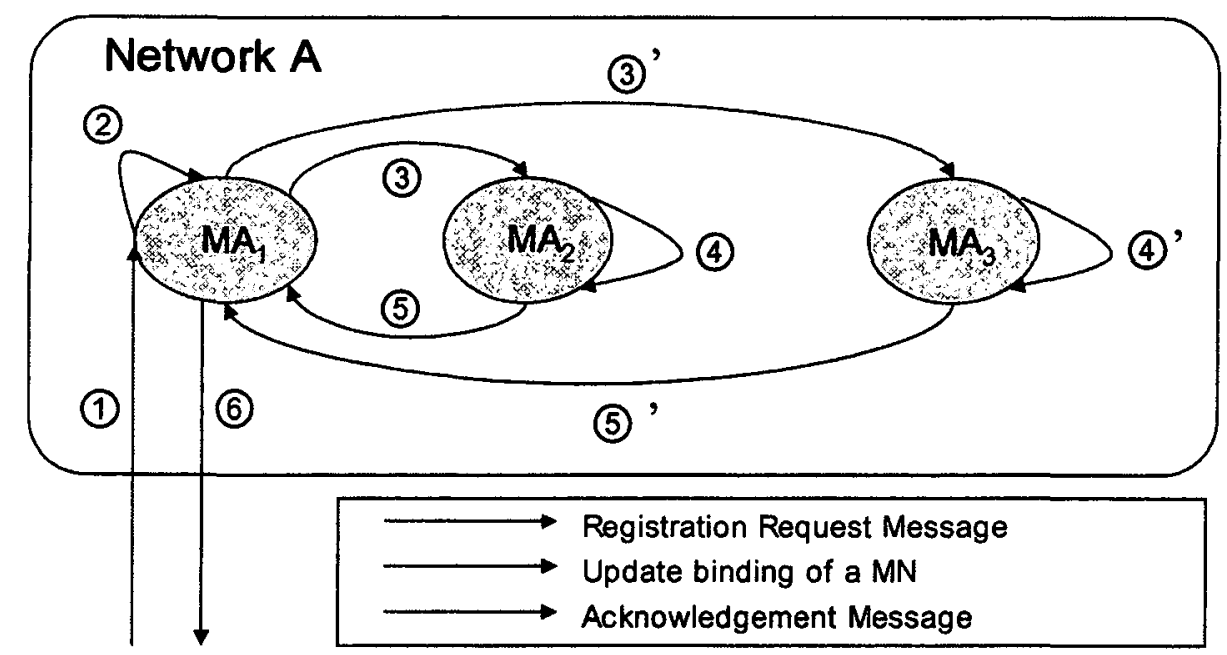

Figure 2 In case of performing a registration process in the PRT schemes

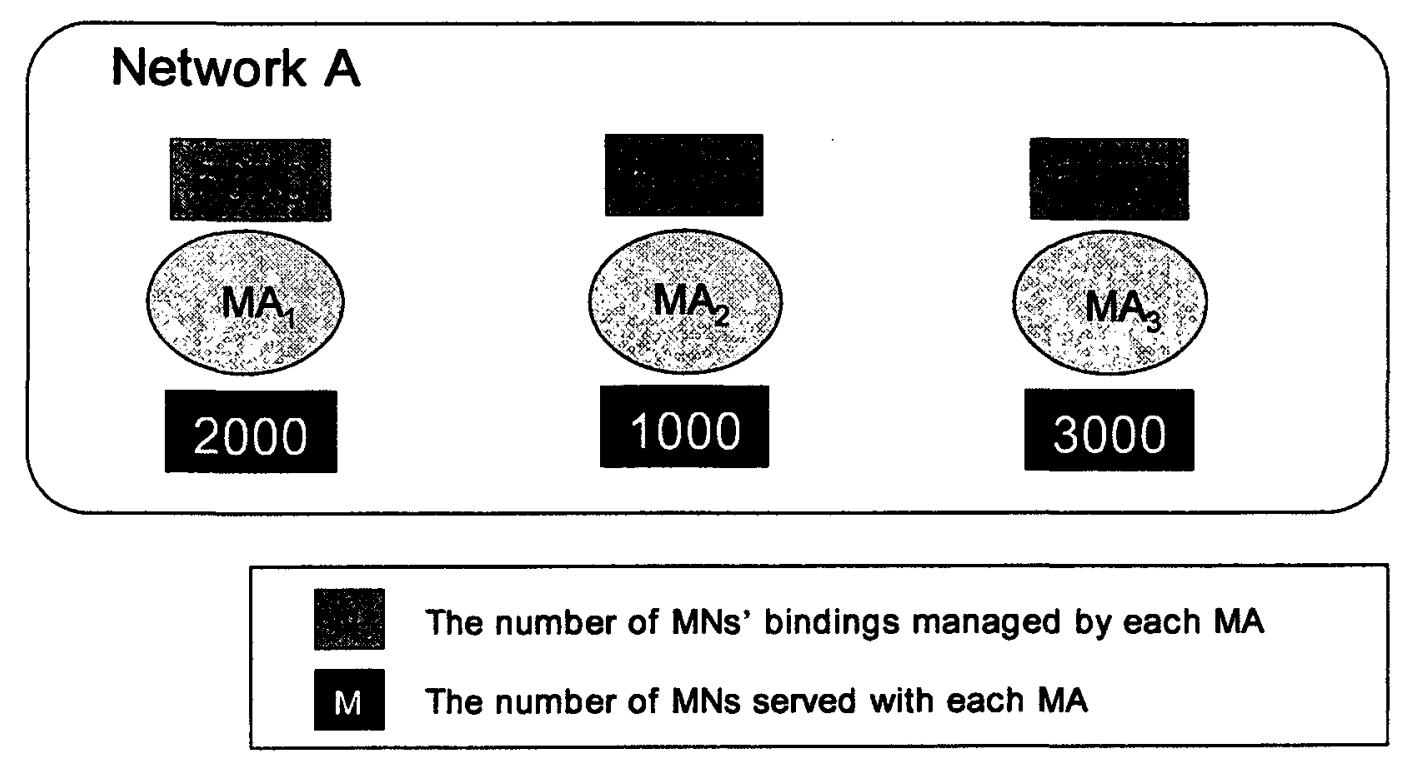

Figure 3 In case of scalability problems of the PRT schemes 
THE CML SCHEME

\section{Basic Idea}

To solve the stated problems of the PRT schemes in this paper, we present the CML scheme using checkpointing and receiver-based pessimistic message logging techniques. For example, suppose $H A_{l}$ is the home agent of $M N_{l}$ and $M N$, currently obtains a care-of address from $F A_{l}$ on the foreign network as in figure 1 . In this case, $M N_{l}$ must send a registration request message to $H A$, through $F A_{\text {l }}$. Receiving the message, $H A_{\text {, }}$ authenticates the message. If the message is invalid, $H A_{l}$ sends $M N_{l}$ a registration reply message for rejection. Otherwise, $H A_{l}$ concurrently performs the following two executions: logging the message into the stable storage and updating the binding of $M N_{l}$ using the message. After having completed both executions, it sends $M N_{l}$ a registration reply message for acceptance. This step ensures that even if $H A$, fails, one among other home agents on the network, named $H A_{i}$, can recover the bindings of all the mobile nodes registering with $H A_{I}$ by restoring the logged registration request messages from the stable storage and replaying them. Moreover, each home agent should periodically save the bindings of all the mobile nodes registering with it on the stable storage and remove all the logged messages beyond the previous checkpoint from the stable storage. Therefore, the CML scheme can reduce the failure-free overhead compared with the PRT scheme presented in [Ghosh (1998)] because in the CML scheme, each MA need not forward each registration request message to the other MAs and wait for each an acknowledgement message from them. Furthermore, the CML scheme improves the scalability to a large number of mobile nodes registering with each network compared with the PRT scheme because each home or foreign agent need to maintain the bindings of only the mobile nodes registering with it.

In Mobile IP, each MA broadcasts an agent advertisement message via its wired or wireless network interface every few seconds. Therefore, in the CML scheme, each MA detects if other MAs fail or not by monitoring their agent advertisement messages. For example, if $H A_{1}$ in network $B$ fails in figure 1 , live HAs on the network can detect its failure because they may receive no agent advertisement message from it. In the CML scheme, one among the live MAs, namely $H A_{j}$, which currently has the minimum number of registering mobile nodes, takes over $H A_{l}$. This step ensures that the CML scheme is scalable even during takeover compared with the PRT schemes using passive replication techniques. $H A_{j}$ restores the bindings of all the mobile nodes registering with $H A_{1}$ and obtains the logged messages for $H A$, from the stable storage. Then, it can recover the latest bindings of all the MNs, which $H A_{l}$ has managed before it failed, by replaying the messages in receive sequence order. After that, $H A_{j}$ performs a gratuitous address resolution protocol mapping $H A_{j}$ 's IP address to $H A_{j}$ 's hardware address to take over $H A_{\text {, }}$ [Plummer (1982)]. Then, $H A_{j}$ serves the MNs on behalf of $H A_{1}$. Therefore, the CML scheme provides the MNs with transparency of their MA's failure and replacement.

If a failed MA, named $M A_{i}$, is repaired in the CML scheme, it should monitor any agent advertisement message, including its IP address, for a few seconds and perform a gratuitous address resolution protocol mapping its IP address to its hardware address. If no other MA has taken over the role of the repaired agent, it should restore the bindings of all the MNs managed before it failed and obtain its logged messages from the stable storage. Then, it can recover the latest bindings of the MNs served in its pre-failure state by replaying the messages in receipt sequence order. If it receives an agent advertisement message including its IP address from a live MA, named $M A_{k}$, it should require from $M A_{k}$ the bindings of the MNs served in its pre-failure state. If $M A_{k}$ fails during its recovery, $M A_{i}$ can recover the latest bindings of the MNs using its checkpoint and logged messages on the stable storage. Therefore, the CML scheme allows each failed MA to recover bindings of the MNs registering with the MA when it is repaired even if all the MAs in the same network fail.

\section{The Description}

In this part, we will first describe our fault-tolerant scheme for home agents and then for foreign agents.

\section{The Description for Home Agents}

\section{(1) Data Structures}

\section{Every home agent in Mobile IP has the following data structures for our scheme.}

- HATable $e_{i}$ It is a vector for saving the timer of each home agent clustered in a network. The timer of each home agent is used so that $H A_{i}$ detects whether the agent is alive or failed currently. The timer of each home agent is initialized to $I N I T$ TIME.

- $R S N_{i}$ : It is the receive sequence number of the latest registration request message which was delivered to $H A_{i}$. It is initialized to 0 .

- Loc_Info: It is a vector for saving the bindings of mobile nodes registering with $H A_{i}$. 
- Log_Info $i$ : It is a set for saving every permitted registration request message delivered to $H A_{i}$ beyond the latest checkpoint and $R S N_{i}$ of the message. Its element is of the form $e=(m s g, r s n)$. It is initialized to $\varnothing$.

(2) Checkpointing and Message Logging Algorithm

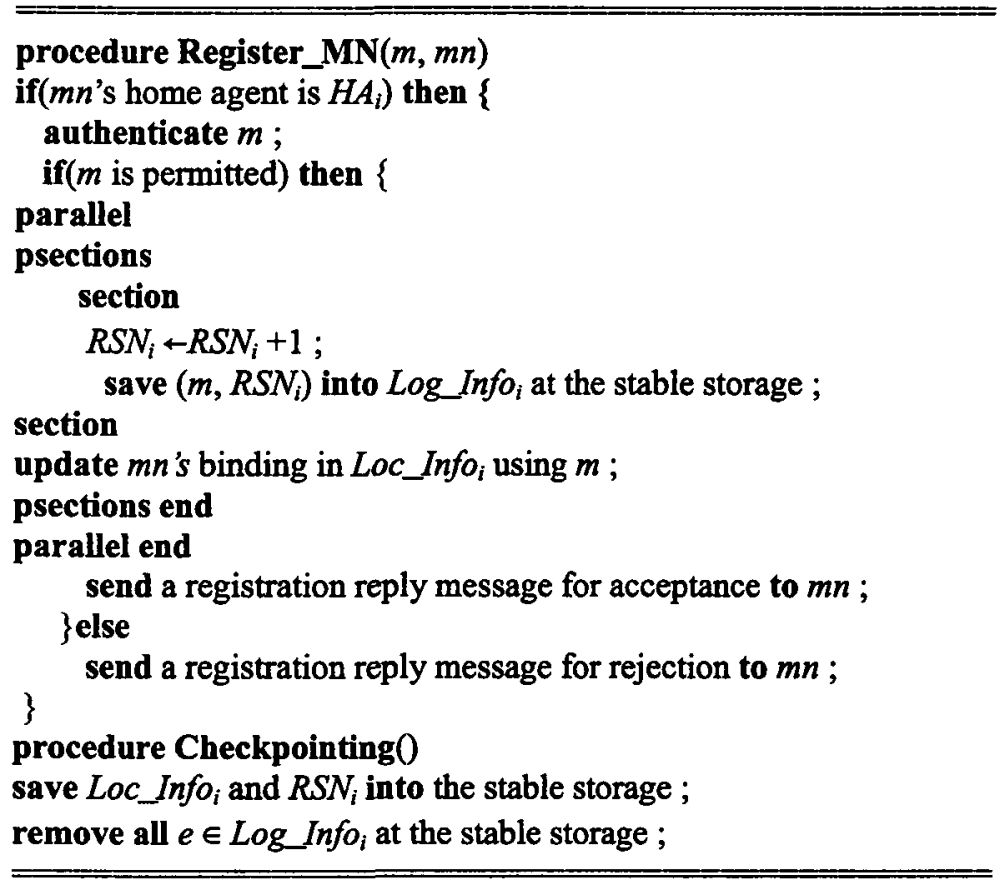

Figure 4 Procedures for $H A_{i}$ 's logging each registration request message during registration process and periodically saving bindings of $M N s$ registering with $H A_{l}$ into stable storage

Figure 4 shows a formal description of our checkpointing and receiver-based pessimistic message logging algorithm. Whenever a home agent $H A_{i}$ receives a registration request message $m$ from a mobile node $m n$, it calls procedure Register_MN(), which first authenticates $m$. If $m$ is valid, $H A_{i}$ performs two executions in parallel: incrementing $R S N_{i}$ by one and saving $\left(m, R S N_{i}\right)$ into Log $\_n f o_{i}$ at the stable storage, and updating $m n$ 's binding in Loc_Info $i$ using $m$. After having completed both executions, it sends $m n$ a registration reply message for acceptance. Otherwise, $H A_{i}$ sends a registration reply message for rejection to $m n$.

If $H A_{i}$ attempts to take its local checkpoint, it calls procedure Checkpointing(). In this procedure, $H A_{i}$ saves Loc_Info $o_{i}$ and its current receive sequence number into the stable storage. Then, it removes all the messages logged in Log_Info ${ }_{i}$ beyond the previous checkpoint.

(3) Failure Detection Algorithm

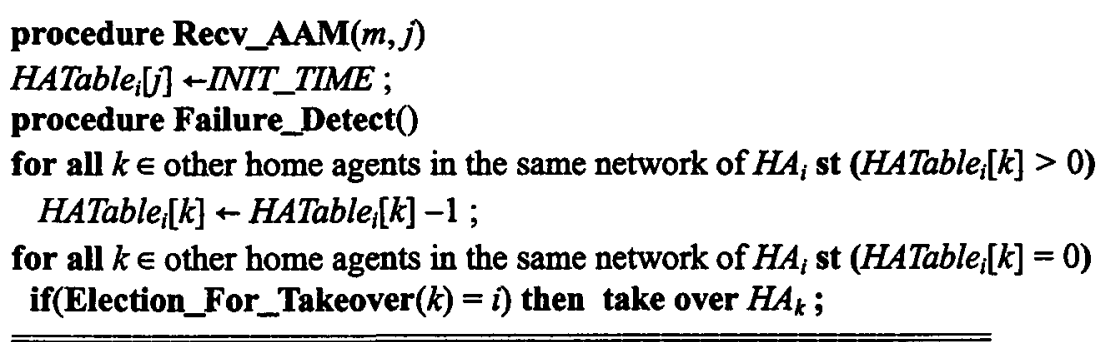

Figure 5 Procedures for $H A_{l}$ 's detecting failures of other home agents in the same network using agent advertisement messages

The home agent failure detection algorithm using agent advertisement messages is given in figure 5. If a home agent $H A_{i}$ receives an agent advertisement message from another home agent $H A_{j}$, it calls procedure Recv_AAM() to set the timer for $H A_{j}$ in HATable $e_{j}$ to $I N I T \_T I M E$.

Whenever ADVERTISING_INTERVAL $(2 \sim 3)$ seconds have elapsed, $H A_{i}$ calls procedure Failure_Detect(). In this procedure, it decrements the timer for every other home agent by one. If among the other home agents in the 
same network, there are ones for which timers expire, procedure Election_For_Takeover() is called so that the remaining home agents determine which live home agents take over the failed ones. In this procedure, among the remaining live agents, one, which serves the minimum number of mobile nodes, takes over a failed agent.

(4) Takeover and Recovery Algorithm

Figure 6 and 7 show a formal description of our takeover and recovery algorithm of a home agent, $H A_{i}$. If $H A_{i}$ takes over a failed agent $H A_{j}$ after having completed Election_For_Takeover(), it calls procedure Take_OverO in figure 6. In this procedure, $H A_{i}$ restores $L o c_{-}$Info $f_{j}, \log \operatorname{Info}_{j}$ and $R S N_{j}$ from the stable storage and then, it updates all the bindings in $L o c \_$Info $f_{j}$ to the latest by replaying each logged message in receive sequence order. Afterward, it performs a gratuitous address resolution protocol mapping $H A_{j}$ 's IP address to its physical hardware address. Then it should serve the MNs having registered with $H A_{j}$, send an agent advertisement message for $H A_{j}$ to other home agents every $A D V E R T I S I N G \_I N T E R V A L$ seconds and respond to every address resolution protocol request message sent for $H A_{j}$.

When a failed home agent $H A_{i}$ is repaired and rebooted, it calls procedure Recover( $)$ in figure 7 . In this procedure, it invokes procedure Listen_To() to listen to any agent advertisement message including its IP address for sometime (i.e., INIT_TIME × ADVERTISING_INTERVAL seconds). Then, it performs a gratuitous address resolution protocol mapping its IP address to its physical hardware address. If it receives no agent advertisement message including its IP address, it restores $L o c \_I n f o_{i}, \log I$ Info $o_{i}$ and $R S N_{i}$ from the stable storage and then updates all the bindings in Loc_Info $o_{i}$ using $\log \_$Info $o_{i}$. If it receives an agent advertisement message including its IP address from $H A_{k}$, it requires $L o c \_I n f o_{i}$ and $R S N_{i}$ from $H A_{k}$ by remotely calling procedure Req_Bindings() at $H A_{k}$. If $H A_{k}$ has failed before completing the procedure, $H A_{i}$ restores $L o c \_$Info , $_{i} \log \_$Info $o_{i}$ and $R S N_{i}$ from the stable storage and then updates all the bindings in Loc_lnfo $o_{i}$ using Log_lnfo $o_{i}$

\title{
The Description for Foreign Agents
}

In Mobile IP, failures of foreign agents are less critical than those of home agents. It means that if a mobile node has registered with a foreign agent in a network and the agent fails currently, the mobile node can re-register with another foreign agent in the same network [Perkins (1996b)]. However, to do so, it should send a registration request message to and receive a registration reply message from its home agent through the new foreign agent.

\author{
procedure Take_Over(j) \\ restore $L o c_{\_} \operatorname{Info} o_{j}, \log \operatorname{Info}_{j}$ and $R S N_{j}$ from the stable storage ; \\ for all $e \in \log$ Info in e.rsn order \\ update Loc_Info replaying e.msg ; \\ perform a gratuitous ARP binding $H A_{j}$ 's IP address to $H A_{i}$ 's physical hardware address ;
}

Figure 6 Takeover Procedures for a home agent $H A_{i}$ 


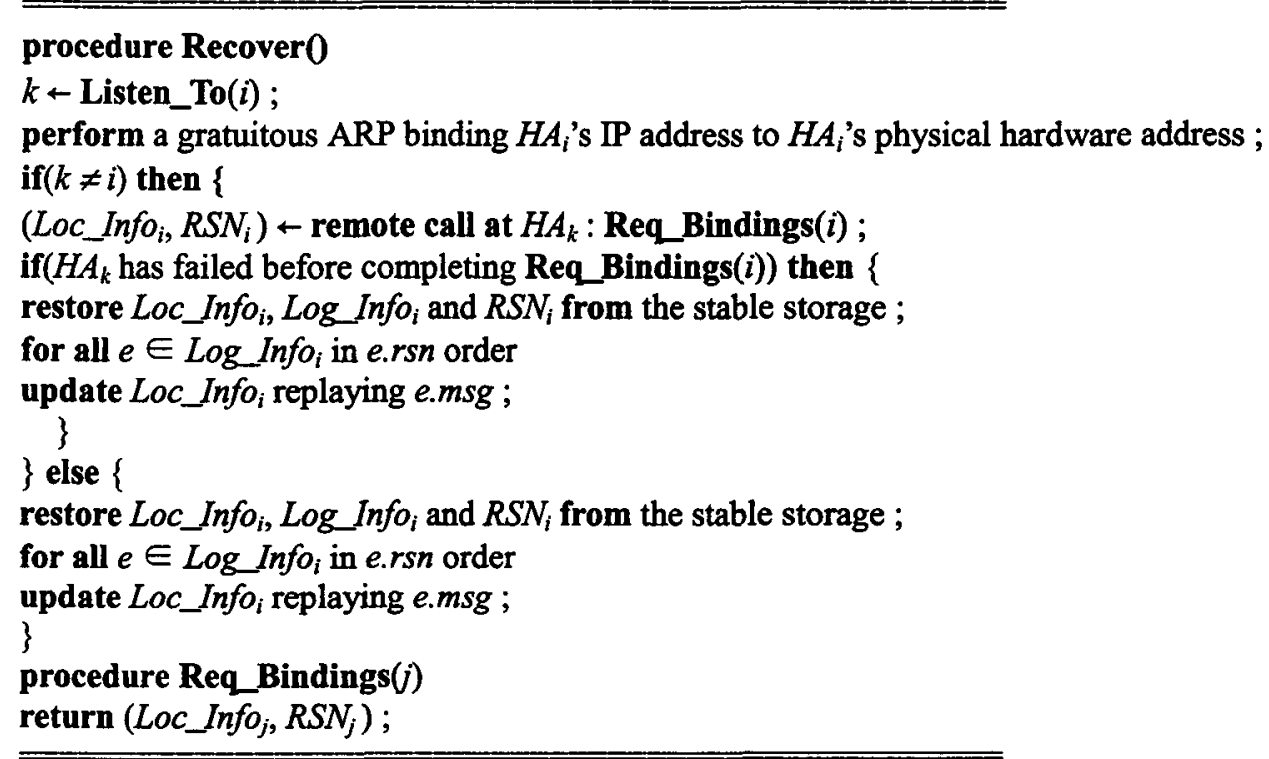

\section{Figure 7 Recovery procedure for a home agent $H A_{i}$}

The re-registration process across the Internet may require high latency for recovering binding of the mobile node. Thus, this method forces other nodes not to communicate with the mobile node until the registration process is completed. To solve the problem of the traditional method, live foreign agents can recover bindings of all the mobile nodes having registered with failed agents faster than the traditional method by using the same scheme as the scheme for home agents described in the previous section. However, unlike a home agent, a foreign agent logs a registration request message, sent from each mobile node having registered with the agent, into the stable storage only after it has received a registration reply message for acceptance from the home agent of the mobile node. If the foreign agent fails, another live foreign agent in the same network restores bindings of the mobile nodes having served by the failed agent and logged messages. In this case, the live foreign agent need not forward each logged message to the corresponding home agent when replaying the message. If the failed foreign agent attempts to recover, but there is no live agent having taken over the failed agent, it replay each logged message in the same manner.

\section{CORRECTNESS}

In this section, we prove the correctness of our checkpointing and message logging algorithms, takeover algorithm and recovery algorithm for mobility agents.

Theorem 1 Our checkpointing and message logging algorithms ensure that the current state of $p, s_{p}{ }^{k}(0 \leq k)$, is recoverable.

Proof: The proof proceeds by induction on $k$, the index of the state interval of each mobility agent $p$.

[Base case]

In this case, $s_{p}{ }^{0}$ is the initial state interval of $p$ and deterministic. Therefore, $s_{p}{ }^{0}$ is trivially recoverable by lemma 2 because $s i_{p}$ is stable.

[Induction hypothesis]

We assume that the theorem is true in case that $k=n$.

[Induction step]

If there is the determinant of $\operatorname{dev}_{p}{ }^{n+1}(m)$ on stable storage because the algorithms allows $s_{p}{ }^{n}$ to be recoverable by induction hypothesis, the theorem is true for $p$ in case that $k=n+1$. In this step, when $p$ receives the $(n+1)$-th registration request message $m$ beyond $s_{p}{ }^{n}$, it saves the determinant of $\operatorname{dev}_{p}{ }^{n+1}(m)$ on stable storage by calling procedure Register_Mn0. Afterwards, if $p$ calls Checkpointing0, it saves $s_{p}{ }^{n+1}$, i.e., the current bindings of the mobile nodes registering with it on stable storage, and then removes all the determinants for $p$ from stable storage. Therefore, our algorithms allow $s_{p}^{n+1}$ to be recoverable.

By the induction, our checkpointing and message logging algorithms ensure that the current state of $p, s_{p}{ }^{k}(0 \leq$ $k$ ), is recoverable.

Theorem 2 If there are $n(2 \leq n)$ redundant mobility agents in a network and even $n$ - 1 among them fail concurrently, all the mobile nodes registering with the failed agents can communicate with other nodes in the system after a live mobility agent has completed our takeover algorithm. 
Proof: The proof proceeds by induction on $n$, the number of redundant mobility agents in a network.

\section{[Base case]}

In this case, there are a failed and a live mobility agent, named $M A_{\text {fall }}$ and $M A_{\text {live }}$, respectively. In our protocol, $M A_{\text {live }}$ detects the failure of $M A_{\text {fail }}$ when $M A_{\text {live }}$ 's timer for $M A_{\text {fail }}$ expires, and then take over $M A_{\text {fail }}$ by executing procedure Election_For_Takeover(). Then, it restores from the stable storage the bindings of all the mobile nodes having registered with $M A_{\text {fail }}$, the logged messages beyond the latest checkpoint for $M A_{\text {fail }}$, and the receive sequence number of the latest logged message delivered to $M A_{\text {fail. }}$. Then, it can recover the latest bindings of the mobile nodes by replaying each logged message in receive sequence number order. Afterward, it performs a gratuitous address resolution protocol mapping $M A_{\text {fail }}$ 's IP address to its physical hardware address. Then it serves the MNs having registered with $M A_{\text {fail }}$, sends an agent advertisement message for $M A_{\text {fail }}$ to other home agents every ADVERTISING_INTERVAL seconds and responds to every address resolution protocol request message sent for $M A_{\text {fail }}$. Thus, $M A_{\text {live }}$ enables the mobile nodes to communicate with other nodes in the system.

[Induction hypothesis]

We assume that the theorem is true in case that $n=k$.

[Induction step]

For $n=k+1$, there are $k$ failed mobility agents and a live one in a network. If the live mobility agent $M A_{\text {live }}$ has only to take over the $k$-th failed mobility agent $M A_{\text {fallk }}$ because $M A_{\text {live }}$ can take over $k$-1 failed mobility agents by induction hypothesis, the theorem is true in case that $n=k+1$.

In our takeover algorithm, $M A_{\text {live }}$ can recover bindings of the mobile nodes having registered with $M A_{\text {failk }}$, and then serve the mobile nodes on behalf of $M A_{\text {fall }}$ in the same manner like base case. Thus, $M A_{\text {live }}$ enables all the mobile nodes having registered with $k$ failed mobility agents to communicate with other nodes in the system.

By the induction, if there are $n$ redundant mobility agents in a network and even $n-1$ among them fail concurrently, all the mobile nodes registering with the failed agents can communicate with other nodes in the system after a live mobility agent has completed our takeover algorithm.

Theorem 3 Our recovery algorithm allows every failed mobility agent to recover the latest bindings of all the mobile nodes served by it before it failed.

Proof: We prove this theorem by contradiction.

Assume that every failed mobility agent cannot recover the latest bindings of all the mobile nodes after having completed our recovery algorithm. When a failed mobility agent $M A_{\text {fail }}$ is repaired and rebooted, it calls procedure Recover(). In this procedure, it listens to any agent advertisement message including its IP address for some seconds and then performs a gratuitous address resolution protocol mapping its IP address to its physical hardware address. There are two cases:

Case 1: There is no live mobility agent having taken over $M A_{\text {fail }}$.

In this case, the information is in the stable storage that is needed for recovering the latest bindings of the mobile nodes served by $M A_{\text {fail }}$. The information consists of bindings of the mobile nodes, logged messages for $M A_{\text {fail }}$ and the receive sequence number of the latest message delivered to its $I P$ address. In Recover $0, M A_{\text {fail }}$ restores the information from the stable storage and then recovers the latest bindings of the mobile nodes by replaying each logged message in receive sequence order.

Case 2: A live mobility agent $M A_{\text {live }}$ has taken over $M A_{\text {fail. }}$. In this case, $M A_{\text {live }}$ has the latest bindings of the mobile nodes served by $M A_{\text {fail }}$. In Recover(), $M A_{\text {fall }}$ requires from $M A_{\text {live }}$ the bindings of all the mobile nodes served by it and the receive sequence number of the latest message delivered to its IP address by remotely calling procedure Req_Bindings 0 at $M A_{\text {live }}$. There are two sub-cases:

Case 2.1: $M A_{\text {live }}$ successfully completes Req_Bindings 0 .

In this case, $M A_{\text {fail }}$ obtains the latest bindings of the mobile nodes from $M A_{\text {live }}$.

Case 2.2: $M A_{\text {live }}$ has failed before it completes Req_Bindings().

In this case, $M A_{\text {fail }}$ recovers the latest bindings of the mobile nodes in the same manner like case 1 .

Hence, both case 1 and 2 contradict the hypothesis.

Table 1. Parameters and their meanings

\begin{tabular}{ll}
\hline Parameter & Meaning \\
\hline NOMN & Number of mobile nodes registering with a network \\
NOMA & Number of redundant mobility agents in a network
\end{tabular}




\section{COMPARISONS}

In this section, we intend to compare the CML scheme with the PRT scheme presented in [Ghosh (1998)] briefly. Generally, performance indices used for evaluation of scalability of the two schemes are the number of MNs whose bindings are managed by each MA in a network (denoted by NOMNMA) and the latency time for its processing a registration request message from each MN (denoted by LTRR). For simplicity, we suppose that each MA in a network serves the same number of MNs. It means that if the number of MNs registering with a network is $n$ and the number of MAs in the network is $m$, the number of MNs served by each agent is $(n / m)$.

First, we evaluate scalability of the two schemes with respect to NOMNMA during failure-free operation. Table 1 shows the parameters, which NOMNMA depends on, and their meanings. If NOMNMAs of the two schemes are calculated using the parameters respectively, NOMNMA of the CML scheme is NOMN / NOMA whereas that of the PRT scheme is $N O M N$ during failure-free operation. Figure 8 illustrates $N O M N M A$ versus $N O M N$ when NOMA is 10. In this figure, NOMNMA increases as NOMN increases in the two schemes. However, we can see that the increasing rate of NOMNMA in the CML scheme is significantly lower than in the PRT scheme. Figure 9 illustrates NOMNMA versus NOMA when NOMN is 6000. As NOMA increases in this figure, NOMNMA of the CML scheme decreases whereas NOMNMA of the PRT scheme is always equal to NOMN. The reason for these results is that each mobility agent in a network must maintain bindings of the mobile nodes registering with all the redundant mobility agents in the same network in the PRT scheme whereas it has only to maintain bindings of the mobile nodes registering with only it in the CML scheme. Therefore, we can see that the CML improves scalability to a large number of mobile nodes managed by each mobility agent compared with the PRT scheme during failure-free operation.

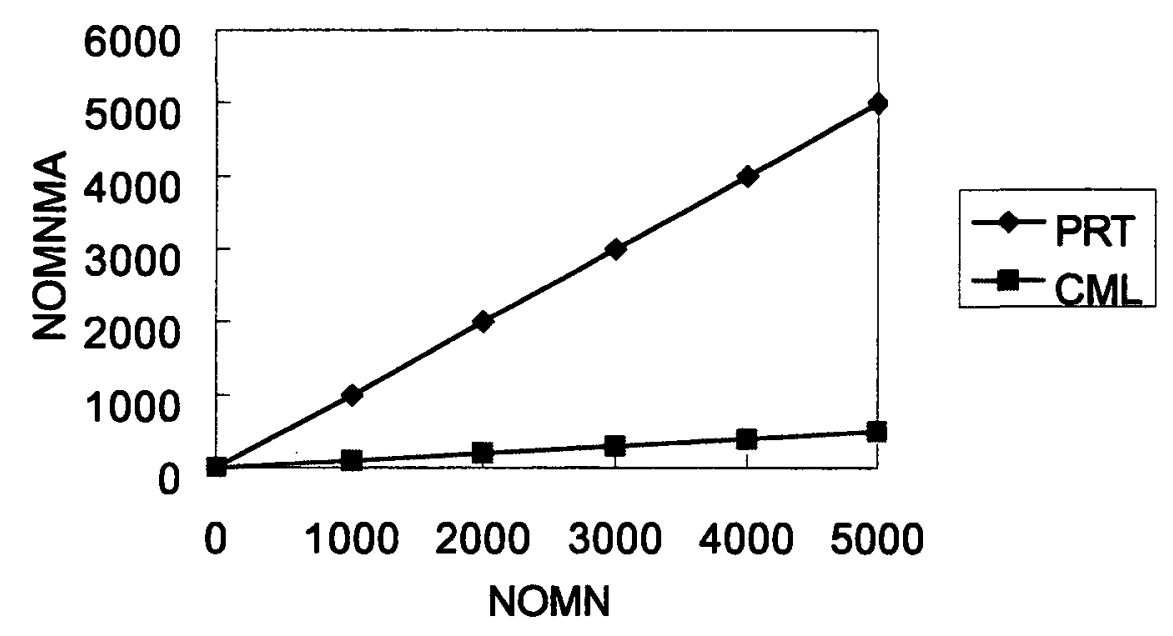

Figure 8 NOMNMA versus NOMN $(N O M A=10)$

Next, we evaluate scalability of the two schemes with respect to LTRR during failure-free operation. If each mobility agent receives a registration request message from a mobile node in the PRT scheme, it should process the message and forward the message to its peers, and wait for receiving all the acknowledgement messages from them. 


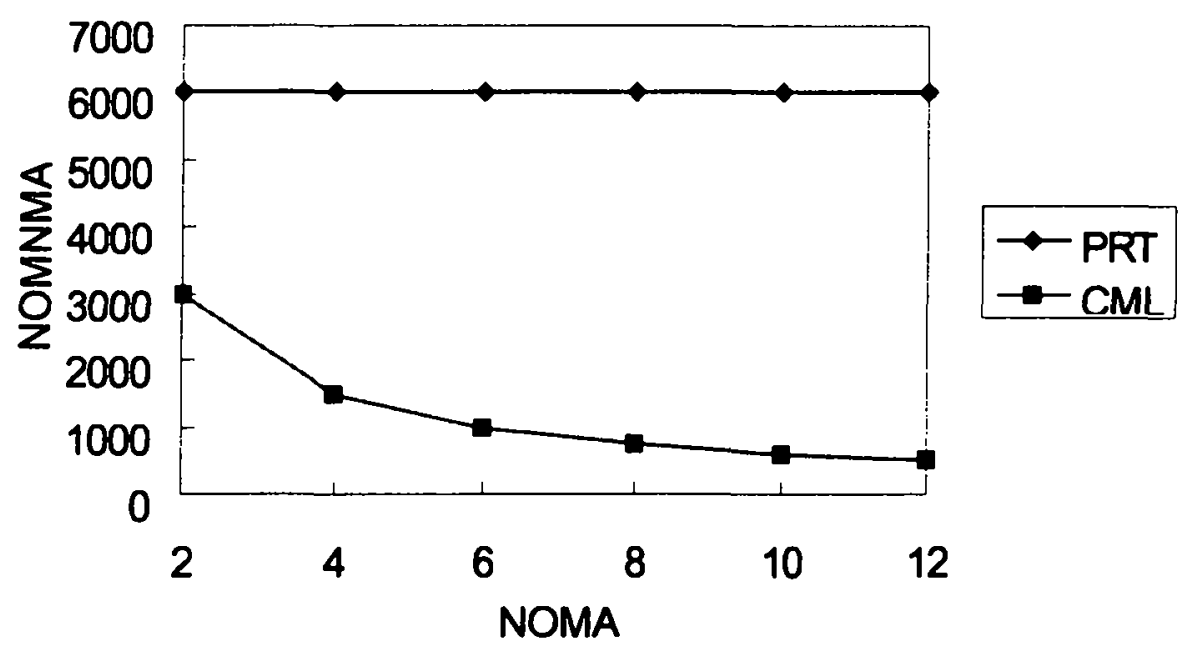

Figure 9 NOMNMA versus NOMA $(N O M N=6000)$

Thus, the total number of messages generated per registration request message in the PRT scheme is $(2 \times$ $(N O M A-1))$ and the number of messages on the critical path is NOMA. However, in the CML scheme, it should process the message and require the stable storage server to save the recovery information of the message into stable storage, and wait for receiving an acknowledgement message from it. Thus, the total number of messages generated per registration request message in the CML scheme is 2 and the number of messages on the critical path is 2 . Therefore, we can see that the total number of messages and the number of messages on the critical path generated per registration request message in the CML scheme, not in the PRT scheme, are always constant. Next, we evaluate overheads of the two schemes for taking over or recovering failed mobility agents. In the PRT scheme, live mobility agents can take over failed agents fast because they always maintain bindings of all the mobile nodes registering with not only itself but also its peers. Therefore, the takeover time of the CML scheme may be longer than that of the PRT scheme because each mobility agent has only to maintain the bindings of the mobile nodes registering with it and live mobility agents should recover the bindings of failed ones from the stable storage. However, the takeover time of the CML scheme can be reduced using two methods. The first method is that each mobility agent maintains only the latest in the stable storage among registration request messages sent from each mobile node registering with the agent. This method decreases the number of logged messages that each live mobility agent should replay when it takes over a failed agent. The second method is implementing the stable storage as a high-speed and scalable storage system such as Storage Area Network (SAN) [IBM (1999)].

If there are live mobility agents in a network, the recovery time of failed and repaired agents are the same in the two schemes because they can recover their bindings from the live mobility agents in the schemes. However, otherwise, each failed agent can recover its latest bindings from the stable storage in the CML scheme whereas it cannot recover them in the PRT scheme.

\section{CONCLUSION}

In this paper, we identified the problems in the PRT schemes using passive replication techniques; high failurefree latency during registration process if the number of MAs in the same network increases and forcing each MA to manage bindings of all the MNs registering with its network. Then, we presented the CML scheme using checkpointing and receiver-based pessimistic message logging techniques. The CML scheme achieves low failure-free latency even if the number of MAs in a network increases, and improves scalability to a large number of MNs registering with each network compared with the PRT schemes. Additionally, the CML scheme allows each failed MA to recover bindings of the MNs registering with the MA when it is repaired even if all the MAs in the same network fail. However, the takeover time of the CML scheme may be longer than that of the PRT scheme like in section 4. The takeover time of the CML scheme can be reduced using the two methods mentioned in section 4 . 


\section{REFERENCES}

Alvisi, L., Hoppe, B. \& Marzullo, K. (1993) "Nonblocking and Orphan-Free Message Logging Protocols", Proc. the $23^{\text {th }}$ Symposium on Fault-Tolerant Computing, pp. 145-154.

Binkley, J. R. \& McHugh, J. (1997) Secure Mobile Networking: Sixth Quarterly Report - Winter 1997, Portland State University.

Dixit, A \& Gupta, V. (1996) Mobile-IP for Linux (ver 1.00), Technical Report, SUNY Binghampton.

Droms, R. (1993) Dynamic Host Configuration Protocol, RFC 1541.

Elnozahy, E. N. \& Zwaenepoel, W. (1992) "Manetho: Transparent rollback-recovery with low overhead, limited rollback and fast output commit", IEEE Transactions on Computers, Vol 41, pp. 526-531.

Elnozahy, E. N., Alvisi, L., Wang, Y. M. \& Johnson, D. B. (1999) A Survey of Rollback-Recovery Protocols in Message-Passing Systems, Technical Report CMU-CS-99-148, Carnegie-Mellon University.

Ghosh, R. \& Varghese, G. (1998) Fault-Tolerant Mobile IP, Technical Report WUCS-98 -11, Washington University.

IBM Corporation and International Data Group. (1999) Survey says Storage Area Networks may unclog future roadblocks to e-Business, News Release.

Johnson, D. B. (1994) "Scalable and Robust Internetwork Routing for Mobile Hosts", Proc. the 14 International Conference on Distributed Computing Systems, pp. 2-11.

Perkins, C. (1996) IP Encapsulation Within IP, RFC 2003.

Perkins, C. (1996) IP Mobility Support, RFC 2002.

Perkins, C. (1996) Minimal Encapsulation With IP, RFC 2004.

Perkins, C. \& Johnson, D. B. (1997) Route Optimization in Mobile-IP, Mobile IP Working Group, Internet Draft.

Plummer, D. C. (1982) An Ethernet Address Resolution Protocol - or - Converting Network Protocol Address to 48 bit Ethernet Address for Transmission on Ethernet Hardware. STD 37, RFC 826.

Postel, J. B. (1981) Internet Protocol, Internet Request For Comments RFC 791.

Rose, M. T. (1990) The Open Book: A Practical Perspective on OSI, Prentice Hall, Englewood Cliffs, NJ.

Schlichting, R. D. \& Schneider, F. B. (1985) "Fail-stop processors: an approach to designing fault-tolerant distributed computing systems", ACM Transactions on Computer Systems, Vol.1, No.3, pp. 222-238.

Simpson, W. (1995) IP in IP Tunneling, RFC 1853.

Solomon, J. D. (1998) Mobile IP, The Internet Unplugged, Prentice Hall Series.

Strom, R. B. \& Yemeni, S. (1985) "Optimistic recovery in distributed systems", ACM Transactions on Computer Systems, Vol.3, No.3, pp. 204-226.

Turner, P. (1990) NetWare communications processes, NetWare Application Notes, Novell Research, pp. 2581. 